\title{
ON THE CONHARMONIC CURVATURE TENSOR OF KENMOTSU MANIFOLDS WITH GENERALIZED TANAKA-WEBSTER CONNECTION
}

\author{
D. G. PRAKASHA AND B. S. HADIMANI
}

Received 14 March, 2015

\begin{abstract}
In this paper, we study a generalized Tanaka-Webster connection on a Kenmotsu manifold. We study the conharmonic curvature tensor with respect to the generalized Tanaka-Webster connection $\widetilde{\nabla}$ and also characterize conharmonically flat and locally $\phi$-conharmonically symmetric Kenmotsu manifold with respect to the connection $\widetilde{\nabla}$. Besides these we also classify Kenmotsu manifolds which satisfy $\widetilde{K} \cdot \widetilde{R}=0$ and $\widetilde{P} \cdot \widetilde{K}=0$, where $\widetilde{K}$ and $\widetilde{P}$ are the conharmonic curvature tensor, the projective curvature tensor and Riemannian curvature tensor, respectively with respect to the connection $\widetilde{\nabla}$.
\end{abstract}

2010 Mathematics Subject Classification: 53C15; 53C25

Keywords: Kenmotsu manifold, generalized Tanaka-Webster connection, conharmonic curvature tensor, Einstein manifold

\section{INTRODUCTION}

In [15], Tanno classified almost contact metric manifold $M$ whose automorphism group attains the maximum dimension. For such a manifold, the sectional curvature of plane section containing $\xi$ is a constant, say $c$. (1) If $c>0, M$ is a homogeneous Sasakian manifold of constant $\phi$-sectional curvature. (2) If $c=0, M$ is global Riemannian product of a line or circle with a Kähler manifold of constant holomorphic sectional curvature. (3) If $c<0, M$ is warped product space $\mathbb{R} \times_{f} \mathbb{C}^{n}$. In 1972, Kenmotsu [9] characterized the differential geometric properties of manifolds of class (3); the structure so obtained is now known as Kenmotsu structure. A Kenmotsu structure is not Sasakian (see [9]). Kenmotsu manifolds have also been studied in several papers $[5,8,11]$ and the references therein.

On the other hand, Tanaka-Webster connection is canonical affine connection defined on a non-degenerate pseudo-Hermition CR-manifold (see [14,17]). The generalized Tanaka-Webster connection for contact metric manifolds by the canonical connection was first studied by Tanno [16]. This connection coincides with the Tanaka-Webster

The first author (DGP) was supported in part by University Grants Commission, New Delhi, India in the form of UGC-SAP-DRS-III programme to the Department of Mathematics, K.U. Dharwad. 
connection if the associated CR-structure is integrable. Acet et al [10] studied a Kenmotsu manifold with respect to the generalized Tanaka-Webster connection.

The paper is organized as follws: After preliminaries, in Section 3, we give a brief account of information regarding the generalized Tanaka-Webster connection $\widetilde{\nabla}$ on Kenmotsu manifolds and obtain some results. In Section 4, we study a conharmonically flat Kenmotsu manifold with respect to the connection $\widetilde{\nabla}$. Section 5 deals with the study of locally $\phi$-conharmonically symmetric Kenmotsu manifold with respect to the connection $\widetilde{\nabla}$. Sections 6 and 7 are devoted to the study of Kenmotsu manifolds with respect to the connection $\widetilde{\nabla}$ satisfying the conditions $\widetilde{K}(\xi, X) \cdot R=0$ and $\widetilde{P}(\xi, X) \cdot \widetilde{R}=0$, respectively.

\section{PReliminaries}

An $n(=2 m+1)$-dimensional differentiable manifold $M$ is called an almost contact Riemannian manifold if either its structural group can be reduced to $U(n) \times\{I\}$ or equivalently, there is an almost contact structure $(\phi, \xi, \eta)$ consisting of a $(1,1)$ tensor field $\phi$, a vector field $\xi$, and 1 -form $\eta$-satisfying

$$
\begin{gathered}
\phi^{2}=-I+\eta \otimes \xi, \\
\eta(\xi)=1, \phi \xi=0 . \eta \cdot \phi=0 .
\end{gathered}
$$

Let $g$ be Riemannian metric compatible with $(\phi, \xi, \eta)$, that is

$$
g(\phi X, \phi Y)=g(X, Y)-\eta(X) \eta(Y)
$$

or equivalently,

$$
g(X, \phi Y)=-g(\phi X, Y) \text { and } g(X, \xi)=\eta(X)
$$

for any vector fields $X, Y$ on $\mathrm{M}$ [2]. If moreover,

$$
\begin{aligned}
\left(\nabla_{X} \phi\right) Y & =-\eta(Y) \phi X-g(X, \phi Y) \xi, \\
\nabla_{X} \xi & =X-\eta(X) \xi,
\end{aligned}
$$

where $\nabla$ denotes the Riemannian connection of $g$ hold, then $(M, \phi, \xi, \eta, g)$ is called an almost Kenmotsu manifold. An almost Kenmotsu manifold becomes a Kenmotsu manifold if

$$
g(X, \phi Y)=d \eta(X, Y) .
$$

In a Kenmotsu manifold $M$, the following relation holds [9]:

$$
\begin{aligned}
\left(\nabla_{X} \eta\right) Y & =g(X, Y)-\eta(X) \eta(Y), \\
R(X, Y) \xi & =\eta(X) Y-\eta(Y) X, \\
R(\xi, X) Y & =\eta(Y) X-g(X, Y) \xi, \\
S(X, \xi) & =-(n-1) \eta(X),
\end{aligned}
$$


where $R$ is the Riemannian curvature tensor and $S$ is Ricci tensor defined by $S(X, Y)=$ $g(Q X, Y)$, where $Q$ is Ricci operator.

A Kenmotsu manifold $M$ is said to be an $\eta$-Einstein manifold if its Ricci tensor $S$ of the form

$$
S=a g+b \eta \otimes \eta,
$$

for some smooth functions $a$ and $b$.

Lemma 1 ([8]). Any $\eta$-Einstein Kenmotsu manifold of dimension $\geq 5$ with $b=$ constant is Einstein.

\section{Generalized Tanaka-Webster connection on a Kenmotsu MANIFOLD}

In the following, we consider the generalized Tanaka-Webster connection $\widetilde{\nabla}$ for a Riemannian manifold $M$ defined by

$$
\widetilde{\nabla}_{X} Y=\nabla_{X} Y+\left(\nabla_{X} \eta\right) Y \cdot \xi-\eta(Y) \nabla_{X} \xi+\eta(X) \phi Y,
$$

for all vector fields $X$ and $Y$, where $\nabla$ is Levi-Civita connection on $M$.

If we use (2.6) and (2.8) in (3.1), we obtain

$$
\widetilde{\nabla}_{X} Y=\nabla_{X} Y+g(X, Y) \xi-\eta(Y) X+\eta(X) \phi Y,
$$

for all vector fields $X$ and $Y$. We call the connection $\widetilde{\nabla}$ defined by (3.2) on a Kenmotsu manifold, the generalized Tanaka-Webster connection on a Kenmotsu manifold.

Let $M$ be an $n$-dimensional Kenmotsu manifold. The curvature tensor $\widetilde{R}$ of $M$ with respect to the connection $\widetilde{\nabla}$ is defined by

$$
\widetilde{R}(X, Y) Z=\widetilde{\nabla}_{X} \widetilde{\nabla}_{Y} Z-\widetilde{\nabla}_{Y} \widetilde{\nabla}_{X} Z-\widetilde{\nabla}_{[X, Y]} Z .
$$

Then, in a Kenmotsu manifold, we have

$$
\widetilde{R}(X, Y) Z=R(X, Y) Z+g(Y, Z) X-g(X, Z) Y,
$$

where $R(X, Y) Z=\nabla_{X} \nabla_{Y} Z-\nabla_{Y} \nabla_{X} Z-\nabla_{[X, Y]} Z$, is the curvature tensor of $M$ with respect to the connection $\nabla$.

The Ricci tensor $\widetilde{S}$ and the scalar curvature $\widetilde{r}$ of the Kenmotsu manifold $M$ with respect to the connection $\widetilde{\nabla}$ is given by

$$
\widetilde{S}(X, Y)=\sum_{i=1}^{n} g\left(\widetilde{R}\left(e_{i}, X\right) Y, e_{i}\right)=S(X, Y)+(n-1) g(X, Y)
$$

and

$$
\widetilde{r}=\sum_{i=1}^{n} \widetilde{S}\left(e_{i}, e_{i}\right)=r+n(n-1),
$$


where $\widetilde{r}$ and $r$ are the scalar curvatures of the connection $\widetilde{\nabla}$ and $\nabla$, respectively. So with the above background, we obtain the following theorem:

Theorem 1. For a Kenmotsu manifold $M$ with generalized Tanaka-Webster connection $\widetilde{\nabla}$,

a) the curvature tensor $\widetilde{R}$ is given by (3.3),

b) the Ricci tensor $\widetilde{S}$ is given by (3.5),

c) $\widetilde{R}(X, Y) Z+\widetilde{R}(Y, Z) X+\widetilde{R}(Z, X) Y=0$,

d) $\widetilde{R}(X, Y, Z, W)+\widetilde{R}(X, Y, W, Z)=0$,

e) $\widetilde{R}(X, Y, Z, W)+\widetilde{R}(Y, X, Z, W)=0$,

f) $\widetilde{R}(X, Y, Z, W)-\widetilde{R}(Z, W, X, Y)=0$,

g) $\underset{\widetilde{S}}{\widetilde{S}}(X, Y) \xi=\widetilde{R}(\xi, X) Y=\widetilde{R}(\xi, X) \xi=0$,

h) $\widetilde{S}(X, \xi)=0$,

i) $\widetilde{r}=r+n(n-1)$,

j) The Ricci tensor $\widetilde{S}$ is symmetric.

Now we begin with the following:

Corollary 1. If a Kenmotsu manifold is Ricci-flat with respect to generalized Tanaka-webster connection, then it is an Einstein manifold.

Proof: The Proof follows immediately from (3.5).

Theorem 2. Let $M$ be a Kenmotsu manifold. If the curvature tensor $\widetilde{R}$ of the generalized Tanaka-Webster connection $\widetilde{\nabla}$ vanishes, then $M$ is locally isomorphic to the hyperbolic space $H^{n}(-1)$.

Proof: Let the curvature tensor $\widetilde{R}$ of the connection $\widetilde{\nabla}$ vanishes. That is, $\widetilde{R}=0$. In view of Eq.(3.4), we have

$$
R(X, Y) Z=g(X, Z) Y-g(Y, Z) X .
$$

This gives

$$
R(X, Y, Z, W)=-[g(Y, Z) g(X, W)-g((X, Z) g(Y, W)] .
$$

This shows that, $M$ is of constant negative curvature -1 .

A space form is said to be hyperbolic if and only if the sectional curvature is negative [3]. Thus, $M$ is locally isometric to the hyperbolic space $H^{n}(-1)$.

Theorem 3. Let $M$ be a Kenmotsu manifold. If $M$ is of constant curvature $c$ with respect to the connection $\widetilde{\nabla}$, then $M$ is of constant curvature $c-1$ with respect to the connection $\nabla$.

Proof: Let $M$ be of constant curvature $c$. Then from (3.3) we have

$$
\widetilde{R}(X, Y, Z, W)=c[g(Y, Z) g(X, W)-g(X, Z) g(Y, W)] .
$$

By taking account of (3.9) in (3.4), it follows that

$$
R(X, Y, Z, W)=(c-1)[g(Y, Z) g(X, W)-g(X, Z) g(Y, W)] .
$$


This shows that, $M$ is of constant scalar curvature $c-1$. This completes the proof.

The notion of a conharmonic curvature tensor was first studied by Ishii [7]. A rank four tensor $K^{\prime}$ that remains invariant under conharmonic transformation for an $n$-dimensional Riemannian Manifold $M$, is given by

$$
\begin{aligned}
K^{\prime}(X, Y, Z, W) & =R^{\prime}(X, Y, Z, W) \\
& -\frac{1}{2 n-1} g(Y, Z) S(X, W)-g(X, Z) S(Y, W) \\
& +S(Y, Z) g(X, W)-S(X, Z) g(Y, W)],
\end{aligned}
$$

where $R^{\prime}$ denotes the Riemannian curvature tensor type $(0,4)$ and $K^{\prime}$ denotes the conharmonic curvature tensor of type $(0,4)$ defined by

$$
\begin{aligned}
R^{\prime}(X, Y, Z . W) & =g(R(X, Y) Z, W), \\
K^{\prime}(X, Y, Z, W) & =g(K(X, Y) Z, W) .
\end{aligned}
$$

where $R$ is the Riemannian tensor of type $(0,3), K$ is the conharmonic curvature tensor of type $(0,3)$ and $S$ denotes the Ricci tensor of type $(0,2)$. The curvature tensor defined by Eq.(3.11) is known as conharmonic curvature tensor [7]. A manifold whose conharmonic curvature vanishes at every point of the manifold is called conharmonically flat manifold. Thus this tensor represents the deviation of the manifold from conharmonic flatness. It satisfies all the symmetry properties of the Riemannian curvature tensor $R$. There are many physical applications of tensor $K$. For example, we refer the readers to see [1]. A conharmonic curvature tensor on a Kenmotsu manifold has been studied in [4].

Analogous to the conharmonic curvature tensor $K$ with respect to Levi-Civita connection $\nabla$, we give the conharmonic curvature tensor $\widetilde{K}$ with respect to generalized Tanaka-Webster connection $\widetilde{\nabla}$.

In a Kenmotsu manifold $M$ of dimension $n>2$, the conharmonic curvature tensor $\widetilde{K}$ with respect to the Tanaka-Webster connection $\widetilde{\nabla}$ is given by

$$
\begin{aligned}
\widetilde{K}(X, Y) Z & =\widetilde{R}(X, Y) Z-\frac{1}{(n-2)} \widetilde{\widetilde{S}}(Y, Z) X-\widetilde{S}(X, Z) Y \\
& +g(Y, Z) \widetilde{Q} X-g(X, Z) \widetilde{Q} Y]
\end{aligned}
$$

for all vector fields $X, Y$ and $Z$ on $M$, where $\widetilde{R}, \widetilde{S}$ and $\widetilde{Q}$ are the Riemannian curvature tensor, Ricci tensor and Ricci operator, respectively with respect to the connection $\widetilde{\nabla}$.

Using (3.4) and (3.5) in (3.14), we get

$$
\widetilde{K}(X, Y) Z=K(X, Y) Z+[g(Y, Z) X-g(X, Z) Y]
$$

or

$$
\widetilde{K}(X, Y) Z=R(X, Y) Z-\frac{n}{n-2}[g(Y, Z) X-g(X, Z) Y]
$$




$$
\begin{aligned}
& -\frac{1}{n-2}[S(Y, Z) X-S(X, Z) Y \\
& +g(Y, Z) Q X-g(X, Z) Q Y] .
\end{aligned}
$$

In a Kenmotsu manifold, using (2.10) and (2.11), the equation (3.16) gives

$$
\begin{aligned}
\widetilde{K}(\xi, Y) Z & =\frac{(n-3)}{(n-2)}[\eta(Z) Y-g(Y, Z) \xi] \\
& +\frac{1}{(n-2)}[\eta(Z) Q Y-S(Y, Z) \xi]=-\widetilde{K}(Y, \xi) Z
\end{aligned}
$$

and

$$
\begin{aligned}
\eta(\widetilde{K}(X, Y) Z) & =\frac{(n-3)}{(n-2)}[g(X, Z) \eta(Y)-g(Y, Z) \eta(X)] \\
& +\frac{1}{(n-2)}[S(X, Z) \eta(Y)-S(Y, Z) \eta(X)]
\end{aligned}
$$

\section{Conharmonically Flat Kenmotsu manifold With ReSPeCt to the CONNECTION $\widetilde{\nabla}$}

A conhormonic curvature tensor $K$ with respect to Levi-Civita connection $\nabla$ is said to be flat if it vanishes identically(that is, $K=0$ ) with respect to the connection $\nabla$. A conharmonically flat Kenmotsu manifold with respect to the semi-symmetric metric connection has been studied in [12].

Assume that, $M$ is conharmonically flat Kenmotsu manifold with respect to the connection $\nabla$. That is, $\widetilde{K}=0$. Then from (3.14), we have

$$
\begin{aligned}
\widetilde{R}(X, Y) Z & =\frac{1}{n-2}[g(Y, Z) \widetilde{Q} X-g((X, Z) \widetilde{Q} Y \\
& +\widetilde{S}(Y, Z) Y-\widetilde{S}(X, Z) X] .
\end{aligned}
$$

This gives,

$$
K(X, Y) Z=[g(Y, Z) X-g(X, Z) Y]
$$

or equivalently,

$$
\begin{aligned}
R(Y, X) Z & =\frac{1}{n-2}[S(Y, Z) X-S(X, Z) Y+g(Y, Z) Q X-g(X, Z) Q Y] \\
& +\frac{n}{(n-2)}[g(Y, Z) X-g(X, Z) Y] .
\end{aligned}
$$

Taking inner product with $W$ in (4.3), then

$$
\begin{aligned}
& R^{\prime}(X, Y, Z, W) \\
= & \frac{1}{(n-2)}[S(Y, Z) g(X, W)-S(X, Z) g(Y, W)+g(Y, Z) S(X, W)
\end{aligned}
$$




$$
-g(X, Z) S(Y, W)]+\frac{n}{n-2}[g(Y, Z) g(X, W)-g(X, Z) g(Y, W)] .
$$

where $R^{\prime}(X, Y, Z, W)=g(R(X, Y) Z, W)$.

Putting $X=W=\xi$ in (4.4) and using (2.10) and (2.11), we have

$$
S(Y, Z)=-(n-1) g(Y, Z) .
$$

Thus, we can state the following:

Theorem 4. A conharmonically flat Kenmotsu manifold $M(n>2)$ with respect to generalized Tanaka-Webster connection $\widetilde{\nabla}$ is an Einstein manifold.

In view of (4.5), (4.3) reduces to

$$
R(Y, X) Z=-[g(Y, Z) X-g(X, Z) Y] .
$$

That is, $M$ is locally isometric to the locally hyperbolic space $H^{n}(-1)$.

On the other hand, If $M$ is locally isometric to the hyperbolic space $H^{n}(-1)$. Then (4.6) holds.

By the virtue of (4.6), (3.2) gives

$$
\widetilde{R}(X, Y) Z=0 .
$$

Similarly, by taking account of (4.5) in (3.5), we have

$$
\widetilde{S}(Y, Z)=0 .
$$

Using (4.7) and (4.8) in (3.14) we obtain

$$
\widetilde{K}(X, Y) Z=0 .
$$

Thus, $M$ is conharmonically flat with respect to the connection $\widetilde{\nabla}$. This leads to the following:

Theorem 5. An n-dimensional Kenmotsu manifols $M(n>2)$ is conharmonically flat with respect to the generalized Tanaka-Webster connection $\widetilde{\nabla}$ if and only if it is locally isometric to the hyperbolic sphere $H^{n}(-1)$.

\section{LOCALLY $\phi$-CONHARMONICALLY SYMMETRIC KENMOTSU MANIFOLDS WITH RESPECT TO THE CONNECTION $\widetilde{\nabla}$}

The notion of locally $\phi$-symmetry was first studied by Takahashi [13] on a Sasakian manifold. In this section we consider a locally $\phi$-conharmonically symmetric Kenmotsu manifolds with respect to the connection $\widetilde{\nabla}$.

Definition 1. An Kenmotsu manifold $M$ is said to be locally $\phi$-conharmonically symmetric with respect to the generalized Tanaka-Webster connection $\widetilde{\nabla}$ if the conharmonic curvature tensor $\widetilde{K}$ with respect to the connection $\widetilde{\nabla}$ satisfies

$$
\phi^{2}\left(\left(\widetilde{\nabla}_{W} \widetilde{K}\right)(X, Y) Z\right)=0,
$$

where $X, Y, Z$ and $W$ are horizontal vector fields on $M$. 
From (3.2), we have that

$$
\begin{aligned}
\left(\widetilde{\nabla}_{W} \widetilde{K}\right)(X, Y) Z & =\left(\nabla_{W} \widetilde{K}\right)(X, Y) Z+g(W, \widetilde{K}(X, Y) Z) \xi \\
& -\eta(\widetilde{K}(X, Y) Z) W+\eta(W) \phi \widetilde{K}(X, Y) Z .
\end{aligned}
$$

Now, Differentiating (3.15) in the direction of $W$, we get

$$
\left(\nabla_{W} \widetilde{K}\right)(X, Y) Z=\left(\nabla_{W} K\right)(X, Y) Z .
$$

Then, using (4.3) and (5.3) in (5.2), we have

$$
\begin{aligned}
& \left(\widetilde{\nabla}_{W} \widetilde{K}\right)(X, Y) Z \\
= & \left(\nabla_{W} K\right)(X, Y) Z+g(W, \widetilde{K}(X, Y) Z) \xi \\
+ & \eta(W) \phi \widetilde{K}(X, Y) Z-\frac{(n-3)}{(n-2)}[g(X, Z) \eta(Y) W-g(Y, Z) \eta(X) W] \\
- & \frac{1}{(n-2)}[S(X, Z) \eta(Y) W-S(Y, Z) \eta(X) W] .
\end{aligned}
$$

Applying $\phi^{2}$ on both sides of (5.4) and using (2.1) and (2.2), we obtain

$$
\begin{aligned}
& \phi^{2}\left(\left(\widetilde{\nabla}_{W} \widetilde{K}\right)(X, Y) Z\right) \\
= & \phi^{2}\left(\left(\nabla_{W} K\right)(X, Y) Z\right)-\eta(W) \phi \widetilde{K}(X, Y) Z \\
+ & \frac{(n-3)}{(n-2)}[g(X, Z) \eta(Y) W-g(Y, Z) \eta(X) W] \\
+ & \frac{1}{(n-2)}[S(X, Z) \eta(Y) W-S(Y, Z) \eta(X) W] \\
- & \frac{(n-3)}{(n-2)}[g(X, Z) \eta(Y) \eta(W) \xi-g(Y, Z) \eta(X) \eta(W) \xi] \\
- & \frac{1}{(n-2)}[S(X, Z) \eta(Y) \eta(W) \xi-S(Y, Z) \eta(X) \eta(W) \xi] .
\end{aligned}
$$

Now, if $X, Y, W$ are horizantal vector fields, then the above equation reduces to

$$
\left.\phi^{2}\left(\left(\widetilde{\nabla}_{W} \widetilde{K}\right)(X, Y) Z\right)=\phi^{2}\left(\nabla_{W} K\right)(X, Y) Z\right) .
$$

This shows that, $M$ is locally conharmonically $\phi$-symmetric with respect to the connection $\widetilde{\nabla}$ if and only if it is so with respect to the connection $\nabla$.

Hence we state the following:

Theorem 6. A Kenmotsu manifold $M(n>3)$ is locally $\phi$-conharmonically symmetric with respect to generalized Tanaka-Webster connection $\widetilde{\nabla}$ if and only if it is so with respect to the Levi-Civita connection $\nabla$. 


\section{KENMOTSU MANIFOLD WITH RESPECT TO THE CONNECTION $\widetilde{\nabla}$ SATISFYING}

$$
\widetilde{K}(\xi, X) \cdot \widetilde{R}=0
$$

In this section consider a Kenmotsu manifold $M$ satisfying the condition

$$
\widetilde{K}(\xi, X) \cdot \widetilde{R}=0
$$

We define $\widetilde{K}(\xi, X) \cdot \widetilde{R}$ by

$$
\begin{aligned}
& (\widetilde{K}(\xi, X) \cdot \widetilde{R})(Y, Z) W \\
= & \widetilde{K}(\xi, X) \cdot \widetilde{R}(Y, Z) W-\widetilde{R}(\widetilde{K}(\xi, X) Y, Z) W \\
- & \widetilde{R}(Y, \widetilde{K}(\xi, X) Z) W-\widetilde{R}(Y, Z) \widetilde{K}(\xi, X) W .
\end{aligned}
$$

From (6.1) and (6.2), we have

$$
\begin{gathered}
(\widetilde{K}(\xi, X) \cdot \widetilde{R})(\xi, Z) W-\widetilde{R}(\widetilde{K}(\xi, X) \xi, Z) W \\
-\widetilde{R}(\xi, \widetilde{K}(\xi, X) Z) W-\widetilde{R}(\xi, Z) \widetilde{K}(\xi, X) W=0 .
\end{gathered}
$$

Using the property $(\mathrm{g})$ of Theorem 3.1 in (6.3), we obtain

$$
\widetilde{R}(\widetilde{K}(\xi, X) \xi, Z) W=0,
$$

which on using (3.17), gives

$$
\frac{(n-3)}{(n-2)} \widetilde{R}(X, Z) W+\frac{1}{(n-2)} \widetilde{R}(Q X, Z) W=0 .
$$

Taking inner-product of (6.5) with $U$ and using (3.4) we get

$$
\begin{aligned}
& \frac{(n-3)}{(n-2)}[R(X, Z, W, U)+g(Z, W) g(X, U)-g(X, W) g(Z, U)] \\
+ & \left.\frac{1}{(n-2)}[R(Q X, Z) W, U)+g(Z, W) S(X, U)-S(X, W) g(Z, U)\right] \\
= & 0 .
\end{aligned}
$$

Let $\left\{e_{i}\right\}(1 \leq i \leq n)$ an orthonormal basis of the tangent space at any point of the manifold. Putting $Z=W=e_{i}$ in (6.6) and taking summation over $i, 1 \leq i \leq n$, we get

$$
S^{2}(X, U)=-2 S(X, U)-(n-1)(n-3) g(X, U) .
$$

This leads to the following:

Proposition 1. In an $n$-dimensional $(n>3)$ Kenmotsu manifold $M$ with respect to the generalized Tanaka-Webster connection $\widetilde{\nabla}$ if the condition $\widetilde{K}(\xi, X) \cdot R=0$ holds on $M$, then the equation (6.7) is satisfied on $M$.

Now we need the following: 
Lemma 2 ([6]). Let A be symmetric $(0,2)$-tensor at a point $X$ of a Semi-Riemannian manifold $\left(M^{n}, g\right),(n>1)$, and let $T=g \bar{\wedge} A$ be the Kulkarni-Nomizu product of $g$ and $A$, Then, the relation.

$$
T \cdot T=\alpha Q(g, T), \alpha \in \mathbb{R}
$$

is satisfied at $x$ if and only if the condition

$$
A^{2}=\alpha A+\lambda g, \quad \lambda \in \mathbb{R}
$$

holds at $x$.

From Proposition 1 and Lemma 2, we have the following:

Corollary 2. Let $M$ be an $n$-dimensional $(n \geq 3)$ Kenmotsu manifold with respect

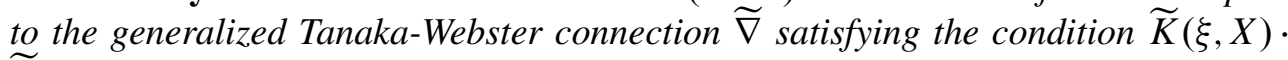
$\widetilde{R}=0$. Then $T \cdot T=\alpha Q(g, T)$, where $T=g \bar{\wedge} A$ and $\alpha=-2$.

\section{KENMOTSU MANIFOLDS WITH RESPECT TO THE CONNECTION $\widetilde{\nabla}$ SATISFYING}

$$
\widetilde{P}(\xi, X) \cdot \widetilde{K}=0
$$

We consider a Kenmotsu manifold $M$ satisfying the condition

$$
\widetilde{P}(\xi, X) \cdot \widetilde{K}=0,
$$

where $\widetilde{P}$ is the projective curvature tensor with respect to the connection $\widetilde{\nabla}$ given by

$$
\widetilde{P}(X, Y) Z=\widetilde{R}(X, Y) Z-\frac{1}{(n-1)}[\widetilde{S}(Y, Z) X-\widetilde{S}(X, Z) Y] .
$$

Now we define $\widetilde{P} \cdot \widetilde{K}$ by

$$
\begin{aligned}
& (\widetilde{P}(\xi, X) \cdot \widetilde{K})(Y, Z) W \\
= & \widetilde{P}(\xi, X) \widetilde{K}(Y, Z) W-\widetilde{K}(\widetilde{P}(\xi, X) Y, Z) W \\
- & \widetilde{K}(Y, \widetilde{P}(\xi, X) Z) W-\widetilde{K}(Y, Z) \widetilde{P}(\xi, X) W .
\end{aligned}
$$

From (7.1) and (7.3) we have

$$
(\widetilde{P}(\xi, X) \cdot \widetilde{K})(Y, Z) W=0 .
$$

For a Kenmotsu manifold $M$, we obtain from (7.2) that

$$
\widetilde{P}(\xi, Y) Z=-\frac{1}{(n-1)} S(Y, Z) \xi-g(Y, Z) \xi .
$$

Taking the inner product with $U$ in (7.4) and using (7.5) we obtain

$$
\begin{gathered}
g(X, \widetilde{K}(Y, Z) W) \eta(U)-g(X, Y) g(\widetilde{K}(\xi, Z) W, U) \\
-g((X, Z) g(\widetilde{K}(Y, \xi) W, U)-g(X, W) g(\widetilde{K}(Y, Z) \xi, Y)
\end{gathered}
$$


ON THE CONHARMONIC CURVATURE TENSOR OF KENMOTSU MANIFOLDS...

$$
\begin{aligned}
& +\frac{1}{(n-1)}[S(X, \widetilde{K}(Y, Z) W) \eta(Y)-S(X, Y) g(\widetilde{K}(\xi, V) W, U) \\
& -S(X, Z) g(\widetilde{K}(Y, \xi) W, U)-S(X, W) g(\widetilde{K}(Y, Z) \xi, U)]=0 .
\end{aligned}
$$

Taking $Z=\xi$ in (7.6), we have

$$
\begin{aligned}
& g(X, \widetilde{K}(Y, \xi) W) \eta(U)-g(X, W) g(\widetilde{K}(Y, \xi) \xi, U) \\
+ & \frac{1}{(n-1)}[S(X, \widetilde{K}(Y, \xi) W) \eta(U)-S(X, W) g(\widetilde{K}(Y, \xi) \xi, U)]=0 .
\end{aligned}
$$

Using (3.17) in (7.7), we get

$$
\begin{aligned}
& \frac{(n-3)}{(n-2)}\left[g(X, Y) \eta(U) \eta(W)+\frac{1}{(n-1)} S(X, Y) \eta(U) \eta(W)\right. \\
+ & g(X, W) \eta(U) \eta(Y)-g(X, W) g(U, Y) \\
+ & \left.\frac{1}{(n-1)} S(X, W) \eta(U) \eta(Y)-\frac{1}{(n-1)} S(X, W) g(U, Y)\right] \\
+ & \frac{1}{(n-2)}\left[S(X, Y) \eta(U) \eta(W)+\frac{1}{(n-1)} S(Q X, Y) \eta(U) \eta(W)\right. \\
- & S(U, Y) g(X, W)-(n-1) g(X, W) \eta(U) \eta(Y) \\
- & \left.\frac{1}{n-1} S(X, W) S(U, Y)-S(X, W) \eta(U) \eta(Y)\right]=0,
\end{aligned}
$$

where $S(Q X, Y)=S^{2}(X, Y)$.

Let $\left\{e_{i}\right\} \quad(1 \leq i \leq n)$ be orthonormal basis of the tangent space at any point. Then the sum for $(1 \leq i \leq n)$ of the relation (7.8) for $X=W=e_{i}$ gives

$$
\begin{aligned}
& (r+n(n-1))\left[\frac{-1}{(n-2)} S(U, Y)-\left(\frac{n-3}{n-2}\right) g(U, Y)+\frac{-2}{n-2} \eta(U) \eta(Y)\right] \\
= & 0 .
\end{aligned}
$$

This implies, either $r+n(n-1)=0$ or

$$
S(Y, U)=-(n-3) g(Y, U)-2 \eta(Y) \eta(U) .
$$

If $r+n(n-1)=0$, then from (3.6) we have $\widetilde{r}=0$.

Next, if the equation(7.10) holds, then in the view of Lemma 1, $M$ is an Einstein manifold. Hence, we state that the following:

Theorem 7. Let $M$ be an $n$-dimensional $(n>3)$ Kenmotsu manifold with respect to the generalized Tanaka-Webster connection $\widetilde{\nabla}$ satisfying the condition $\widetilde{P}(\xi, X)$. $\widetilde{K}=0$. Then either $\widetilde{r}=0$, that is, the scalar curvature with respect to the connection $\widetilde{\nabla}$ vanishes or $M$ is an Einstein manifold. 


\section{ACKNOWLEDGEMENT}

The authors are thankful to the referee for his/her valuable suggestions towards the improvement of this paper. Also, the first author (DGP) is thankful to University Grants Commission, New Delhi, for financial support in the form of UGC-SAP-DRSII Programme to the Department of Mathematics, Karnatak University, Dharwad.

\section{REFERENCES}

[1] D. Abdussattar, "On conharmonic transformations in general relativity," Bulletin of the Calcutta Mathematical Society, vol. 41, pp. 409-416, 1966.

[2] D. E. Blair, Contact manifolds in Riemannian geometry, Lecture Notes in Mathematics, Vol. 509. Springer-Verlag, Berlin-New York, 1976.

[3] B. Y. Chen, Geometry of submanifolds, Pure and Applied Mathematics, No. 22. Marcel Dekker, Inc., New York, 1973.

[4] K. De and U. C. De, "Conharmonic curvature tensor on Kenmotsu manifolds," Bulletin of the Transilvania University of Brasov• Vol, vol. 6, no. 55, 2013.

[5] U. C. De and G. Pathak, "On 3-dimensional Kenmotsu manifolds," Indian Journal of Pure and Applied Mathematics, vol. 35, no. 2, pp. 159-166, 2004.

[6] R. Deszcz, L. Verstrelen, and S. Yaprak, "Warped products realizing a certain condition of pseudosymmetry type imposed on the Weyl curvature tensor," Chinese Journal of Mathematics, pp. 139-157, 1994.

[7] Y. Ishii, "On conharmonic transformations," Tensor, NS, vol. 11, pp. 73-80, 1957.

[8] J.-B. Jun, U. C. De, and G. Pathak, "On Kenmotsu manifolds," J. Korean Math. Soc, vol. 42, no. 3, pp. 435-445, 2005, doi: 10.4134/JKMS.2005.42.3.435. [Online]. Available: https://doi.org/10.4134/JKMS.2005.42.3.435

[9] K. Kenmotsu, "A class of almost contact Riemannian manifolds," Tohoku Mathematical Journal, Second Series, vol. 24, no. 1, pp. 93-103, 1972, doi: 10.2748/tmj/1178241594. [Online]. Available: https://doi.org/10.2748/tmj/1178241594

[10] S. Y. Perktaş, B. E. Acet, and E. Kiliç, "Kenmotsu manifolds with generalized tanaka-webster connection," Adiyaman University Journal of Science, vol. 3, no. 2, pp. 79-93, 2013.

[11] G. Pitis, "Geometry of Kenmotsu manifolds," Publishing House of Transilvania University of Brasov, Brasov, vol. 160, 2007.

[12] D. G. Prakasha, A. T. Vanli, C. S. Bagewadi, and D. A. Patil, "Some classes of Kenmotsu manifolds with respect to semi-symmetric metric connection," Acta Mathematica Sinica, English Series, vol. 29, no. 7, pp. 1311-1322, 2013, doi: 10.1007/s10114-013-0326-1. [Online]. Available: https://doi.org/10.1007/s10114-013-0326-1

[13] T. Takahashi, "Sasakian $\varphi$-symmetric spaces," Tohoku Mathematical Journal, Second Series, vol. 29, no. 1, pp. 91-113, 1977, doi: 10.2748/tmj/1178240699. [Online]. Available: https://doi.org/10.2748/tmj/1178240699

[14] N. Tanaka, "On non-degenerate real hypersurfaces, graded Lie algebras and Cartan connections," Japanese journal of mathematics. New series, vol. 2, no. 1, pp. 131-190, 1976.

[15] S. Tanno, "The automorphism groups of almost contact Riemannian manifolds," Tohoku Mathematical Journal, Second Series, vol. 21, no. 1, pp. 21-38, 1969, doi: 10.2748/tmj/1178243031. [Online]. Available: https://doi.org/10.2748/tmj/1178243031

[16] S. Tanno, "Variational problems on contact Riemannian manifolds," Transactions of the American Mathematical Society, vol. 314, no. 1, pp. 349-379, 1989, doi: 10.2307/2001446. [Online]. Available: https://doi.org/10.2307/2001446 
[17] S. M. Webster, "Pseudo-Hermitian structures on a real hypersurface," Journal of Differential Geometry, vol. 13, no. 1, pp. 25-41, 1978, doi: 10.4310/jdg/1214434345. [Online]. Available: http://projecteuclid.org/euclid.jdg/1214434345

\section{Authors' addresses}

\section{G. Prakasha}

Department of Mathematics, Karnatak University, Dharwad - 580003, India

E-mail address: prakashadg@email.com, dgprakasha@kud.ac.in

\section{B. S. Hadimani}

Department of Mathematics, Karnatak University, Dharwad - 580003, India.

Current address: Department of Mathematics, Manipal Institute of Technology, Manipal Academy of Higher Education, Manipal - 576104, India.

E-mail address: balachandrash@gmail.com, bs.hadimani@manipal.edu 